\title{
Low back pain in a teenager rare cause - Morquios syndrome
}

\author{
Subramanian Nallasivan ${ }^{1, *}$, Radha Mahadevan ${ }^{2}$ \\ ${ }^{1}$ Consultant, ${ }^{2}$ Associate Professor, ${ }^{1}$ Velammal Medical College Hospital \& Research Institute, Madurai, Tamil Nadu, ${ }^{2}$ Tamil \\ Nadu Government Multi Super Speciality Hospital, Chennai, Tamil Nadu, India
}

*Corresponding Author:

Email: drsubramanian14@gmail.com

Teenage children presenting with back pain is unusual. Back pain due to musculoskeletal cause varies from mechanical pathologies, juvenile spondyloarthritis, biomechanical strain and metabolic causes like osteomalacia/rickets. Skeletal dysplasia is uncommon in general population and metabolic bone diseases usually manifest in childhood. Rare genetic diseases are increasingly identified because of definite genetic studies and inheritance.

Mucopolysaccharidosis is one among the metabolic bone diseases where genetic defect causes enzyme deficiency thereby resulting in accumulation of glycosaminoglycans in the cell, accounting for the skeletal and systemic manifestations. Unrecognized patients may mimic as juvenile spondyloarthritis or JIA. Hence it's important to recognise these unusual features but mimics as classic patients with arthritis, which would help the patient enormously.

\section{Case History}

A 16 year old boy was seen with back and hip pain for few weeks. He denied any injury and no inflammatory symptoms. He attended with a limping gait. On further history he revealed about the family history of 3 siblings suffering with gene problem and her sister (24 years old) who was the index patient gave detailed information. (Her medical records had documentation as below)

She had painful hips at age 8 and her $\mathrm{x}$ ray showed anterior beaking of vertebral bodies and MRI showed platyspondyly. Her immunologies at that time including CRP, B27, CCP, CBC, calcium all were normal. She also had muscle biopsy which was normal and synovial biopsy of knees did not show any evidence of inflammation. In view of her skeletal picture and back pain, she underwent metabolic screen and her urine glycosaminoglycans was positive for mucopolysaccharides. There was no evidence of Wilson's disease. She was born to non consanguineous parents and has 2 sisters and one brother of which 2 of them share this disease in common.

The boy was evaluated with imaging and metabolic screen. Apart from $x$ rays showing the definite evidence of Morquios syndrome, (Images attached) he also had inflammatory markers checked (normal), and low Vitamin d levels. His urine glycosamino glycans was normal which is the norm in this age.

\section{Discussion}

Lysosomal storage disorders are caused by deficiency of enzymes catalyzing the degradation of glycosaminoglycans (GAGs). They are characterized by intralysosomal accumulation and increased excretion in urine of partially degraded GAGs, - causing organ dysfunction. MPSs are transmitted in an autosomal recessive fashion, except for MPS II, which is X-linked. Morquios syndrome (MPS IV) manifests with odontoid hypoplasia, pectus carinatum, Kyphoscoliosis and dwarfism. X rays- flared Ilium, squared vertebra, beaking borders, and platyspondyly.

Skeletal dysplasia is characterized by incomplete ossification and successive imbalance of growth, which results in a prominent forehead, abnormal face with a large mandible, short neck, cervical spinal cord compression, tracheal obstruction, pectus carinatum, hypermobile joints and other features. Intellectual impairment does not occur.

Odontoid hypoplasia is the most critical skeletal feature to be recognized in any patient with Morquio syndrome. This condition, in combination with ligamentous laxity and extradural mucopolysaccharide deposition, results in atlantoaxial subluxation, leading to consequential quadriparesis or even death.

Individuals with untreated severe Morquio A syndrome die of respiratory obstruction, cervical spinal cord complications, or heart valve disease in their second or third decade of life. The lifespan of individuals with the attenuated form of Morquio A syndrome has been noted to be as long as 70 years. ${ }^{3}$ The height prognosis is poor among individuals with severe bone dysplasia. Intellectual impairment does not occur in Morquio A syndrome.

Cardiovascular manifestations of Morquio syndrome include coronary heart disease and valve thickening manifesting as cardiac dysfunction. Heart disease is clinically evident in adults with Morquio syndrome. Mild mitral or aortic valvular disease is common, and myocardial thickening, systemic and pulmonary hypertension, and narrowing of the coronary arteries with ischemia are rare. The aortic valvular disease is more likely to occur in Morquio A syndrome. ${ }^{[4]}$ Cardiac evaluation at regular intervals with echocardiography is useful in the treatment of patients via serial monitoring of ventricular function and size. 


\section{Differential Diagnoses}

1. Hunter Syndrome (Mucopolysaccharidosis Type II

2. Sanfilippo Syndrome (Mucopolysaccharidosis Type III)

3. Maroteaux-Lamy (Mucopolysaccharidosis Type VI

Syndrome

4. Sly Syndrome (Mucopolysaccharidosis Type VII

5. GM1 Gangliosidosis

Clinical recognition of unique skeletal abnormalities, combined with radiographic and biochemical analyses, is important in the diagnosis of Morquio syndrome (mucopolysaccharidosis type IV [MPS IV]). Although radiographic findings provide substantial insight, they need to be combined with biochemical analysis (enzyme activity), and molecular analysis to diagnose Morquio syndrome.

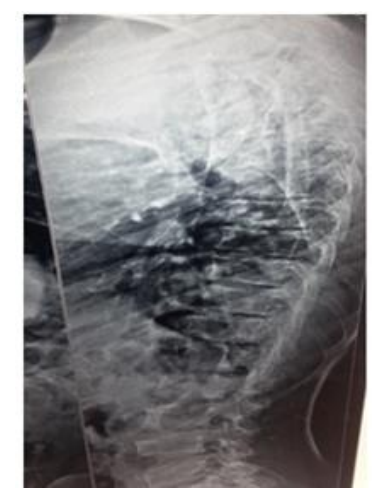

Fig. 1: X ray Lumbar spine- Beaked vertebra and Scoliosis

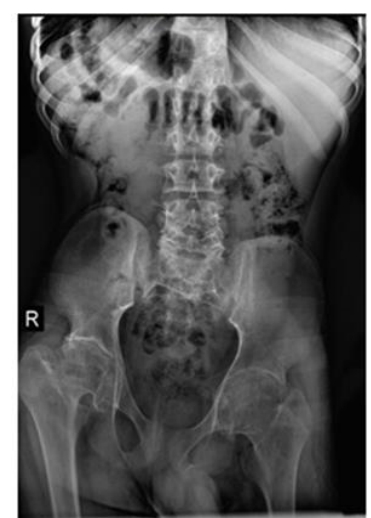

Fig. 2: X ray Spine- Scoliosis and osteoarthritis hips

Imaging: Radiography, CT, and MRI are used to evaluate compression and instability in the upper cervical spine and spinal stenosis. These imaging studies commonly reveal anterior beaking, kyphoscoliosis, platyspondyly, and vertebrae irregularity. Imaging before age 2 years is important. Patients should be monitored annually to determine whether orthopedic surgery is required.

A major complication is cervical myelopathy. A history of exercise intolerance in patients with Morquio syndrome often precedes occult cervical myelopathy, which can also cause bowel and bladder dysfunction and compression of the spinal cord, leading to weakness or paralysis. Mortality and morbidity are primarily related to the atlantoaxial instability and subsequent cervical myelopathy.

Bone marrow transplant and enzyme replacement therapy (ERT) have some success. ${ }^{1,2}$ Our patient also had low vitamin D and being replaced. Her current hip and back pain are due to mechanical reasons and low vitamin D which is so common in India, though it's a tropical country.

\section{Conclusion}

Back and hip pain in children may be due to metabolic cause and diligent evaluation remains the key to unfold the mystery in rare diseases.

Funding: No funding sources.

Conflict of interest: None declared.

\section{References}

1. A. Rodriguez, A. J. Espejo, A. Hernandez et al., "Enzyme replacement therapy for Morquio A: an active recombinant $\mathrm{N}$-acetylgalactosamine-6-sulfate sulfatase produced in Escherichia coli BL21," J Industrial Microbiol Biotechnol 2010;37(11):1193-1201.

2. Shunji Tomatsu et al. Morquio A Syndrome: Diagnosis and Current and Future Therapies. Pediatr Endocrinol Rev 2014;12(01):141-51.

3. Tomatsu S, Montaño AM, Oikawa H, Smith M, Barrera L, Chinen Y, et al. Mucopolysaccharidosis type IVA (Morquio A disease): clinical review and current treatment. Curr Pharm Biotechnol 2011;12(6):931-45.

4. Kampmann C, Abu-Tair T, Gökce S, Lampe C, Reinke J, Mengel E, et al. Heart and Cardiovascular Involvement in Patients with Mucopolysaccharidosis Type IVA (Morquio-A Syndrome). PLoS One 2016;11 (9):e0162612.

How to cite this article: Nallasivan S., Mahadevan R. Low back pain in a teenager rare cause - Morquios syndrome. IP Int J Orthop Rheumatol 2018;4(2):83-4. 